\title{
A randomized controlled trial to prevent glycemic relapse in longitudinal diabetes care: Study protocol (NCT00362 I 93)
} Mary Margaret Huizinga2,3,12, Ayumi Shintani ${ }^{4}$, Stephanie Michon², Anne Brown 1,5, Kathleen Wolff1,5, Laurie Shackleford2, Elaine Boswell King1,5, Rebecca Pratt Gregory ${ }^{1}$, Dianne Davis ${ }^{1}$, Renee Stiles ${ }^{2}$, Tebeb Gebretsadik ${ }^{4}$, Kong Chen ${ }^{6,7,8,10}$, Russell Rothman ${ }^{1,2}$, James W Pichert ${ }^{9}$, David Schlundt ${ }^{11}$ and Tom A Elasy*1,2,3,12

Address: ${ }^{1}$ Diabetes Research and Training Center, Vanderbilt University Medical Center, Nashville, TN, USA, ${ }^{2}$ Division of General Internal Medicine and Public Health, Department of Medicine, Center for Health Services Research, Vanderbilt University Medical Center, Nashville, TN, USA, ${ }^{3}$ VA Tennessee Valley Healthcare System, GRECC, Nashville, TN, USA, ${ }^{4}$ Department of Biostatistics, Vanderbilt University Medical Center, Nashville, TN, USA, 5 School of Nursing, Vanderbilt University Medical Center, Nashville, TN, USA, ${ }^{6}$ Division of Gastroenterology, Department of Medicine, Vanderbilt University Medical Center, Nashville, TN, USA, ${ }^{7}$ Department of Biomedical Engineering, Vanderbilt University Medical Center, Nashville, TN, USA, ${ }^{8}$ Department of Surgery, Vanderbilt University Medical Center, Nashville, TN, USA, ${ }^{9}$ Center for Patient and Professional Advocacy, Vanderbilt University Medical Center, Nashville, TN, USA, ${ }^{10}$ Energy Balance Laboratory, Vanderbilt University Medical Center, Nashville, TN, USA, ${ }^{11}$ Department of Psychology, Vanderbilt University, Nashville, TN, USA and ${ }^{12}$ VA National Quality Scholars Program, Nashville, TN, USA

Email: Mary Margaret Huizinga - mary.margaret.huizinga@vanderbilt.edu; Ayumi Shintani - ayumi.shintani@vanderbilt.edu; Stephanie Michon - stephanie.michon@vanderbilt.edu; Anne Brown - anne.brown@vanderbilt.edu; Kathleen Wolff - kathleen.wolff@vanderbilt.edu; Laurie Shackleford - laura.shackleford@vanderbilt.edu; Elaine Boswell King - elaine.boswell.king@vanderbilt.edu; Rebecca Pratt Gregory - becky.gregory@vanderbilt.edu; Dianne Davis - dianne.davis@vanderbilt.edu; Renee Stiles - renee.stiles@vanderbilt.edu; Tebeb Gebretsadik - tebeb.gebretsadik@vanderbilt.edu; Kong Chen - kong.chen@vanderbilt.edu; Russell Rothman - russell.rothman@vanderbilt.edu; James W Pichert - jim.pichert@vanderbilt.edu; David Schlundt - david.schlundt@vanderbilt.edu; Tom A Elasy* - tom.elasy@vanderbilt.edu

* Corresponding author

Published: 20 October 2006

Implementation Science 2006, I:24
Received: 10 August 2006

Accepted: 20 October 2006

This article is available from: http://www.implementationscience.com/content///I/24

(c) 2006 Huizinga et al; licensee BioMed Central Ltd.

This is an Open Access article distributed under the terms of the Creative Commons Attribution License (http://creativecommons.org/licenses/by/2.0), which permits unrestricted use, distribution, and reproduction in any medium, provided the original work is properly cited.

\begin{abstract}
Background: Diabetes is a common disease with self-management a key aspect of care. Large prospective trials have shown that maintaining glycated hemoglobin less than $7 \%$ greatly reduces complications but translating this level of control into everyday clinical practice can be difficult. Intensive improvement programs are successful in attaining control in patients with type 2 diabetes, however, many patients experience glycemic relapse once returned to routine care. This early relapse is, in part, due to decreased adherence in self-management behaviors.

Objective: This paper describes the design of the Glycemic Relapse Prevention study. The purpose of this study is to determine the optimal frequency of maintenance intervention needed to prevent glycemic relapse. The primary endpoint is glycemic relapse, which is defined as glycated hemoglobin greater than $8 \%$ and an increase of $1 \%$ from baseline.

Methods: The intervention consists of telephonic contact by a nurse practitioner with a referral to a dietitian if indicated. This intervention was designed to provide early identification of self-care problems, understanding the rationale behind the self-care lapse and problem solve to find a negotiated solution. A total of 164 patients were randomized to routine care (least intensive), routine care with phone contact every three months (moderate intensity) or routine care with phone contact every month (most intensive).
\end{abstract}


Conclusion: The baseline patient characteristics are similar across the treatment arms. Intervention fidelity analysis showed excellent reproducibility. This study will provide insight into the important but poorly understood area of glycemic relapse prevention.

\section{Background}

Diabetes is a common disease and has great impact on the individual and society[1]. The burden of diabetes is expected to increase as the population ages, becomes more ethnically diverse and more obese[2]. Self-management of diabetes is critical to prevent the complications associated with diabetes and, yet, remains difficult for many patients to sustain.

Recent large randomized controlled trials have proven that tight glycemic control reduces the microvascular and macrovascular complications of diabetes [3-5]. Reduction of these complications also leads to a great cost savings to healthcare and society[6]. However, it has been difficult to translate the success of these large randomized control trials to everyday practice [7-9]. A recent cross-sectional analysis of 95 clinicians revealed only $40.5 \%$ of type 2 diabetes patients had a glycated hemoglobin (HbA1c) less than 7\%[9]. Even large, well-conducted, multi-factorial randomized controlled trials aimed at reducing $\mathrm{HbA1c}$ have not had success in maintaining long-term glycemic control[10]. The disparity of care between the large trials and a primary care office is largely due to the difference in resources available in the typical medical office. Practical, sustainable ways of maintaining tight glycemic control are needed in everyday practice. Indeed, a number of for profit corporations have entered this arena of disease management given a seeming inability of the current clinical milieu to adequately address this issue.

While diabetes improvement programs are successful in acutely lowering HbA1c [11-24] the long-term effectiveness of these programs is disappointing. Approximately $40 \%$ of those who return to routine care after completing an intensive diabetes improvement program experience a relapse in their glycemic control within one year [25-27]. While some of the glycemic relapse may represent a natural progression of the underlying disease, it is unlikely that such a high percentage would experience such significant disease progression in such a short period of time[4,28]. Some proportion of the relapse is likely due to a patient's inability to maintain adherence to key self-care behaviors - diet, exercise, self-monitoring of blood glucose and medication regimen. Little is known about the optimal frequency, intensity or nature of maintenance interventions needed to prevent deterioration of self-care behaviors that lead to glycemic relapse.

\section{Hypothesis}

The purpose of this study is to better understand prevention of glycemic relapse. The primary aim of this study is to assess the relative effectiveness of three management approaches, varying in frequency, for preventing glycemic relapse after glycemic control has been achieved through participation in an intensive diabetes improvement program. This study will determine the optimal frequency of intervention needed to prevent glycemic relapse in patients with type 2 diabetes. The authors hypothesize that high intensity intervention will lead to a decrease in glycemic relapse in a dose dependent fashion.

Other aims to be addressed in this study include determination of patient characteristics and behaviors predictive of glycemic relapse. In doing so, specific subgroups in need of alternative maintenance strategies will also be identified. Finally, this study will also determine the differences in activity cost between the intervention arms using activity based accounting.

\section{Methods Study Design}

This study is a prospective, randomized control trial to assess the relative effectiveness of three management strategies for the purpose of preventing glycemic relapse in type 2 diabetes. The subjects will be randomized to one of three arms: routine follow-up in a primary care clinic (control), telephone contact every three months (moderate intensity) or telephone contact every month (high intensity). The duration of the study is 24 months. At the completion of the intervention period, the subjects will be asked to complete another 12 months of follow-up during which everyone will receive routine care only. The primary endpoint is glycemic relapse. Glycemic relapse is defined as a HbA1c greater than $8 \%$ and an increase by $1 \%$ point from baseline. The primary analysis will be based on intention to treat.

\section{Study Setting}

Telephonic intervention based out of an academic center in middle Tennessee. At recruitment, study participants lived in the city and surrounding suburbs of the academic center.

\section{Study Population}

All subjects are recruited after completion of a 12 week outpatient, intensive diabetes improvement program following referral for poor glycemic control (HbA1c>8\%). 
The intensive improvement program consists of instruction and support in diabetes self-management coupled with intensification of glycemic medications, including insulin. It is provided by nurse practitioners and supervised by a practicing diabetologist. The educational content includes diet, exercise, self-monitoring of blood glucose and medication adherence as well as instruction in preventive measures such as foot care and screening for complications. Upon completion of the program, only those subjects referred to the improvement program for poor glycemic control (HbA1c>8\%) and who obtained control $(\mathrm{HbA} 1 \mathrm{c}<8 \%$ and at least an absolute $1 \%$ decline in HbA1c) during the program were recruited. Only subjects aged 18-75 years of age were included. Pregnant women were excluded.

\section{Randomization}

Two weeks after completion of the improvement program, a research assistant contacted patients and gave them a brief explanation of the study. The subjects were then invited to participate in the study if they met the defined inclusion criteria. A research assistant confirmed eligibility. After informed consent was obtained, patients were randomly assigned to one of three study arms. Randomization applied permuted block scheme for balancing interval, varying randomly among 3, 6, 9 and 12 according to the outcome of a computer generated random number. This ensured that the cumulative number of assignments to each treatment would be in balance after each block of assignments had been made. The allocation sequence was written by the statistician involved with the trial. Once treatment arm status was assigned by the research assistant, subjects in the intervention arms were assigned a study nurse practitioner. Due to the nature of this intervention, blinding of participants, investigators and study nurse practitioners was not possible. See Figure 1 for enrollment and randomization scheme.

\section{Intervention}

The intervention consists of a phone contact by a nurse practitioner with a referral to a dietitian if nutrition selfcare is perturbed. The characteristics of the intervention are described in Table 1 using a diabetes intervention taxonomy previously characterized[29] The duration of each contact was monitored. During the first session, shared goal setting was established and referred to or modified during subsequent contacts. The method and content of the phone contacts varied based on the assessment. If there were no problems related to glycemic control or selfcare behaviors identified, then Protocol 1 was followed (see Figure 2). If a problem was identified, Protocol 2 was followed (see Figure 2). The intervention does not vary between the treatment arms; only the frequency of the intervention varies.

Protocol 1 is characterized by anticipatory planning for potential lapses, including practicing a coping skill, and also offers self-efficacy enhancement through positive reinforcement, short-term goal setting and cognitive rewards. If a self-care problem was identified then protocol 2 was followed. The subject was asked to identify the source of the struggle. If readily identified, the interviewer employed a 5 step problem solving paradigm: 1) Define problem clearly, 2) Brainstorm strategies, 3) Choose a strategy, 4) Develop an action plan and 5) Try it and revise as needed. If a subject was unable to identify a reason for deteriorating self-care behavior, motivational interviewing was employed largely as a diagnostic modality[30] Subjects were asked to assess the importance of and their confidence in correcting the lapse behavior. The individ-

Table I: Intervention Structure

\begin{tabular}{ll}
\hline Setting & One-on-One \\
Delivery & Phone contact \\
Teaching Methods & Shared goal setting \\
& Problem solving \\
& Cognitive re-framing \\
& Diaries \\
Content & Diet \\
& Exercise \\
& Self-monitoring of blood glucose \\
& Medication management \\
Provider & Diabetes certified nurse educator with a dietician referral if diet self-care is perturbed \\
Tailoring of intervention to an assessment & Yes \\
Modification of intervention with follow-up & Yes \\
Intensity of intervention & \\
Number of episodes & Arm 2: 8 \\
& Arm 3: 24 \\
Duration of episodes & Measured as part of study protocol \\
Duration of study & 24 months \\
Initial supplement given & No \\
\hline
\end{tabular}




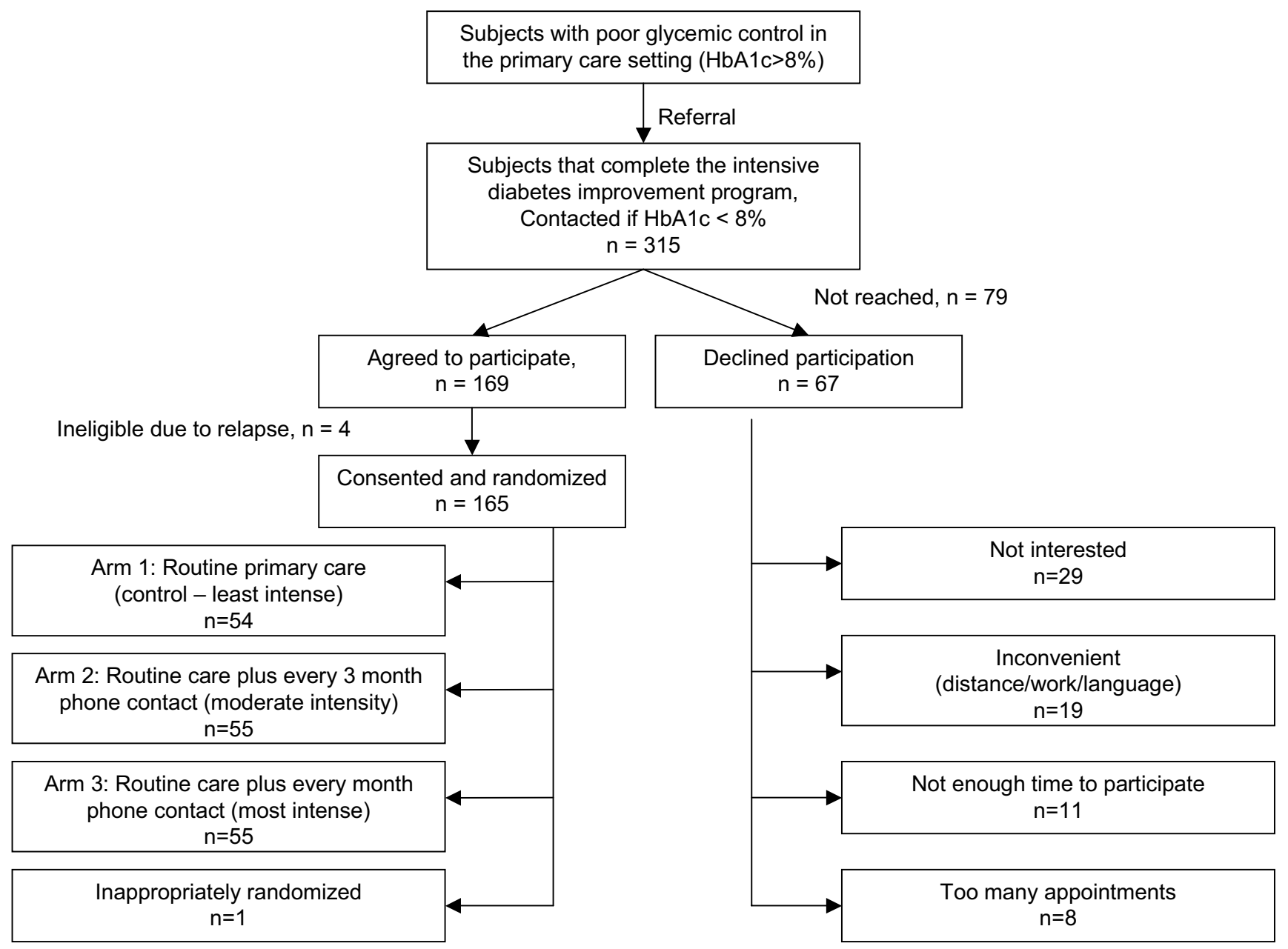

\section{Figure I}

Enrollment and Randomization.

ual was then asked to comment on what prevented them from giving a higher importance/confidence score. This often identified an underlying problem which led back to the problem solving paradigm. In addition to providing a diagnostic tool for identification of the reasons behind the lapse, the motivational interview may also provide a cue to action via the subject's reflection during the assessment.

The interviewer worked with the subject to correct (e.g. correcting a cognitive distortion) the underlying reason for the perturbation of the self-care behavior. If the obstacle could not be corrected (i.e. divorce, financial barrier), then the interviewer worked with the subject to develop a coping mechanism. However, if the subject remained unable to identify a reason for lapse in self-care behavior or to devise a coping strategy, the interviewer worked with the subject to negotiate a change in another self-care behavior as a compensation for the perturbed behavior. A negotiated compensation, for example, may include increased exercise, increased monitoring or increased insulin use for a perturbation of diet self-care.

\section{Intervention Fidelity}

To enhance the reliability and validity of the behavioral intervention portion of this study, intervention fidelity tools were used to monitor the phone contacts between the nurse practitioners and the study subjects [31-33]. The analysis consisted of qualitative descriptions of the extent to which a sample of intervention phone calls was consistent with the intervention protocol (Figure 2) and guidelines. Consistency between the nurse practitioners was also determined. Raters used checklists derived from the protocols to document which elements were conducted or omitted. Overall, adherence to the protocol was quite high with almost all elements present in more than $80 \%$ of all interviews. The educators did not differ significantly in any category. 


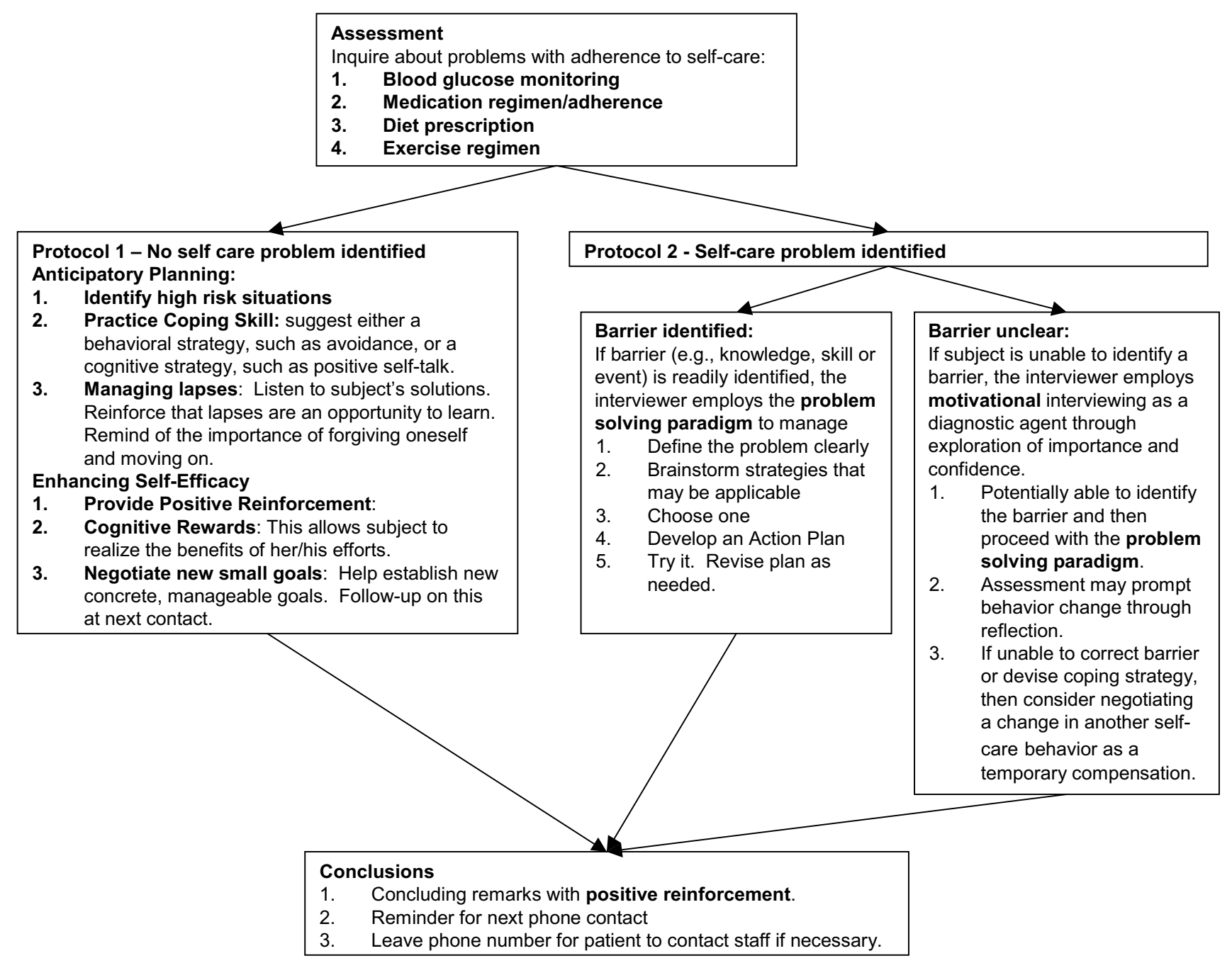

Figure 2

Telephone Contact Intervention Flow Sheet.

\section{Primary Outcome}

The primary outcome is the glycemic relapse rate at 24 months. Relapse is defined as a HbA1c greater than $8 \%$ and an absolute 1\% increase from baseline. The HbA1c will be measured at baseline and at 6-month intervals throughout the study.

\section{Secondary Outcomes Activity Assessment}

A pager-sized $(2.8 \times 2.2 \times 1.1$ inches, weighing $2.3 \mathrm{oz})$ triaxial portable accelerometer (RT3 Research Activity Tracker by StayHealthy, Inc. Monrovia CA) is used to measure detailed movements in the center of body mass (worn at the hip). The RT3 monitor is programmed with each study participant's weight, height, age, and gender prior to application. During each of the visits, each subject is fitted with the RT3 monitor securely on his/her right hip, either by direct clipping to the belt or using a small pouch-bag (for women who do not usually wear a waist belt). Subjects are instructed to wear the RT3 monitor during all possible non-sleeping activities, except during water sports, for the next 7 days. Once the monitor is initialized, it runs continuously without interruption from the subject (no buttons to push). At the end of the 7-day monitoring period, the RT3 is mailed back to the study coordinator via a pre-addressed/stamped bubble envelope and its data downloaded. Using the raw activity counts and a prediction model which was previously developed and validated [34], the total energy expenditure and overall physical activity levels during each study period are obtained for each subject. Furthermore, utilizing durations of activities within certain intensity categories (utilizing the minute-to-minute measurements), subject's adherence to exercise will be validated. 


\section{Depression Score}

The Center for Epidemiologic Studies Depression Scale (CES-D) is used to assess depression in this study. The CES-D is a well-validated, 20 item self-administered questionnaire that quantifies the frequency of depressive symptoms over the previous 7 days. Four items are reversed scored and the total possible score is 60 with $0-$ 9 representing no to minimal symptoms, 10-16 mild symptoms, 17-24 moderate symptoms and >24 severe depressive symptoms.[35]

\section{Cost Accounting Analysis}

Cost analysis of the interventions will be assessed using activity based cost (ABC) accounting techniques[36]. ABC differs from conventional cost accounting in that $A B C$ establishes a causal relationship between work performed, the costs thereof, and the clinical outcomes of the same. In so doing, $\mathrm{ABC}$ enables researchers to quantify more precisely the costs of interventions, the skill level of the team member performing the task, the sequence of activities, and the patients' outcomes.

\section{Data Management}

Data is entered into MS Access (Microsoft Corporation, Redmond, WA) tables. Management report generating programs are used to track subject's progress through the study and to generate letters when visits are due. This also allows for early identification of missing data.

\section{Study size}

Sample size calculation was performed based on chisquare test for linear trend in proportions of patients among the three study arms who relapsed during the study period (118). We expected $50 \%$ of patients who are assigned to study arm A (routine primary care follow-up) relapse during the study period, 30\% in the study arm B (scheduled 3 month interaction with a certified nurse), and $20 \%$ in the study arm C (scheduled 1 month interaction with a certified nurse). Anticipating 20\% attrition, 165 subjects (55 recruited/44 complete study) will provide $85 \%$ power to detect statistically significant linear trend at 2-sided 5\% alpha level. Calculations for power analysis were performed by using nQuery Advisor version 4.0 (Statistical Solutions, Stonehill Corporate Center, Saugus, MA).

\section{Ethics}

This trial received approval from the Vanderbilt Institutional Review Board. An information sheet was given to all subjects and those who agreed to participate were consented prior to randomization. Informed consent was obtained from all subjects. Subjects are free to withdraw from the study at any time, although they were encouraged to decline randomization unless they were prepared to participate in the study for 24 months. The confidenti- ality of the study data are maintained as follows: once computerized, data are not linked to identifying information and the original documents are kept in locked cabinets. The computerized records are identified by study number which is the only link to the subject's identification. Access to the identifying information is restricted to the principal investigator and the study coordinator. Patients received $\$ 50$ upon completion of the study.

\section{Population characteristics}

Enrollment started June 2002 and concluded in January 2005. A total of 164 subjects completed randomization. The control group consists of 54 subjects and each of the intervention arms consists of 55 patients. The baseline characteristic were similar across the groups, see Table 2, with no statistically significant differences.

The average age $( \pm$ SD) of the population was $55 \pm 10.7$ years. Forty-four percent were female and $20 \%$ were African-American. The average HbA1c $( \pm$ SD) was $6.7 \pm 0.68$ and the average duration of diabetes $( \pm$ SD) was $7.1 \pm 8.2$ years. Fifty-four percent used insulin with a median of 55 (IQR 25-92) units/day of insulin. The average BMI ( \pm SD) was $34 \pm 6.9 \mathrm{~kg} / \mathrm{m}^{2}$ and the average waist circumference $( \pm$ $\mathrm{SD})$ was $42.9 \pm 5.8 \mathrm{~cm}$. Results for the CES-D were available for 118 subjects and the median CES-D was 9 (IQR 417). The CES-D results were available with equal frequencies in each study arm.

Baseline physical activity data was successfully obtained in 154 subjects. The baseline measures of daily energy expenditure, physical activity level (PAL) and time spent in moderate and vigorous physical activities (MVPA) were similar in all three groups (see Table 2) and fairly similar to average sedentary populations.

The initial nurse's assessment for the intervention groups were similar (see Table 3). The initial assessment occurred within 2 months of the completion of the intensive outpatient diabetes improvement program. The average number of minutes spent on the initial phone contact was $19.6 \pm 9.3$. Five variables were assessed by the nurses including glycemic control, self blood glucose monitoring, medication adherence, diet adherence and exercise adherence. The majority of the patients answered unchanged in each category for this baseline assessment. At baseline, 28\% had already self-reported worsening of their glycemic control since completion of the improvement program.

\section{Discussion}

This study will advance our understanding of maintenance of glycemic control. The authors approached relapse prevention in a novel way - by determining the "dose" of intervention needed to prevent glycemic 
Table 2: Baseline population characteristics

\begin{tabular}{|c|c|c|c|}
\hline Characteristic & Control Group $(n=54)$ & Moderate Intensity Group $(n=55)$ & High Intensity Group $(n=55)$ \\
\hline Age, yrs & $56.2 \pm 10$ & $55.7 \pm 11$ & $53.5 \pm 11$ \\
\hline Female, n (\%) & $23(43)$ & $21(38)$ & $28(5 I)$ \\
\hline African American, $\mathrm{n}(\%)$ & $7(13)$ & $16(29)$ & $12(22)$ \\
\hline$\geq$ High School, n (\%) & $47(87)$ & $49(89)$ & $50(91)$ \\
\hline Duration of diabetes, yrs & $5.5(0.7-10)$ & $4.0(0.5-10)$ & $4.0(0.5-10)$ \\
\hline Insulin use, $\mathrm{n}(\%)$ & $32(54)$ & $49(45)$ & $50(58)$ \\
\hline Units of insulin per day & $39(24-79)$ & $59(32-100)$ & $61(25-93)$ \\
\hline Weight, lbs & $225 \pm 48$ & $215 \pm 37$ & $223 \pm 51$ \\
\hline $\mathrm{BMI}$ & $34 \pm 7$ & $33 \pm 6$ & $35 \pm 7$ \\
\hline Waist circumference, in & $43.5 \pm 6.2$ & $41.8 \pm 4.8$ & $43.3 \pm 6.3$ \\
\hline $\mathrm{HbAlc}$ & $6.7 \pm 0.7$ & $6.6 \pm 0.7$ & $6.8 \pm 0.6$ \\
\hline Systolic BP & $126 \pm 15$ & $125 \pm 17$ & $127 \pm 15$ \\
\hline Diastolic BP & $72 \pm 9$ & $72 \pm 11$ & $73 \pm 12$ \\
\hline Total cholesterol & $177 \pm 28$ & $178 \pm 35$ & $174 \pm 34$ \\
\hline $\mathrm{HDL}$ & $43 \pm 13$ & $44 \pm 11$ & $41 \pm 11$ \\
\hline LDL & $97 \pm 28$ & $97 \pm 30$ & $98 \pm 31$ \\
\hline Triglycerides & $185(124-229)$ & $168(124-246)$ & $161(112-219)$ \\
\hline CES-D & $9(4-18)$ & $10(4-17)$ & $7(4-14)$ \\
\hline DEE & $3007 \pm 671$ & $2963 \pm 659$ & $3097 \pm 869$ \\
\hline PAL & $1.31 \pm 0.08$ & $1.32 \pm 0.08$ & $1.34 \pm 0.09$ \\
\hline MVPA & $62(35-91)$ & $61(40-116)$ & $77(4 I-126)$ \\
\hline
\end{tabular}

Reported as mean \pm standard deviation or median (interquartile range).

$\mathrm{n}$ - number; BMI - body mass index ( $\left.\mathrm{kg} / \mathrm{m}^{2}\right)$; HbAlc - glycated hemoglobin (\%); BP - blood pressure; HDL - high-density lipoprotein (mg/dL); LDL - low-density lipoprotein ( $\mathrm{mg} / \mathrm{dL}$ ); DEE - daily energy expenditure (kcal); PAL - physical activity level = total energy expenditure/resting energy expenditure; MVPA - moderate to vigorous physical activity (intensity $>3 \times$ resting energy expenditure) $(\mathrm{min} /$ day)

relapse. The intervention is carefully outlined to allow for reproducibility. Intervention fidelity is excellent. This study will also compare the cost of the intervention to routine care. As there is a burgeoning business in chronic care management, it is important to study chronic care interventions for both efficacy and cost-effectiveness to aid in the development of evidence based services.

Table 3: Baseline nurses' assessment

\begin{tabular}{lcc}
\hline Variable & Moderate Intensity Group $(\mathrm{n}=55)$ & High Intensity Group $(\mathrm{n}=55)$ \\
\hline Length of phone call, min & $21.0 \pm 9.4$ & $18.7 \pm 8.9$ \\
Glycemic control & & $10(19)$ \\
$\quad$ Improved & $12(22)$ & $25(48)$ \\
Unchanged & $30(56)$ & $17(33)$ \\
Worse & $12(22)$ & $1(2)$ \\
Self blood glucose monitoring & & $39(74)$ \\
Improved & $3(6)$ & $13(25)$ \\
Unchanged & $43(81)$ & $1(2)$ \\
Worse & $7(13)$ & $45(85)$ \\
Medication adherence & & $7(13)$ \\
Improved & $1(2)$ & $8(15)$ \\
Unchanged & $46(87)$ & $34(64)$ \\
Worse & $6(11)$ & $11(21)$ \\
Diet adherence & & \\
Improved & $9(18)$ & $14(27)$ \\
Unchanged & $33(65)$ & $26(50)$ \\
Worse & $9(18)$ & $12(23)$
\end{tabular}

Reported as mean \pm standard deviation or $\mathrm{n}(\%)$. 
While little is known about relapse of glycemic control, extrapolation is possible from the practical experience available in the obesity, alcohol and smoking literature. Perri et al demonstrated that routine contact with providers was the only variable predictive of weight loss maintenance[37]. Baum et al found that a 3 month provider supported program resulted in greater maintenance of initial weight loss for 12 months as compared to a control group[38]. To minimize relapse after alcohol treatment, Marlatt recommends a behavioral maintenance package consisting of identification of high-risk situations, training in problem solving, actual practice coping with highrisk situations and development of cognitive coping skills[39]. Baer's cognitive behavior model of the relapse process in smoking puts forth that due to prior poor conditioning, individuals are actively coping with situation specific urges to smoke[40]. To prevent smoking relapse, Baer recommends systematic but brief assessment, encouragement, goal setting, planning for risk, reinterpreting lapses, recommendations for lifestyle changes and follow-up appointments. The study intervention is firmly rooted in health behavior methods and draws from prior experience in other diseases such as obesity, smoking and alcohol. While maintenance of self-care behaviors is critical to prevent glycemic relapse, the "dose" of maintenance intervention needed is unknown.

Limitations of this study include reproducibility of the intervention and the possible differences in the routine care received. While the intervention is outlined in this article, it may be difficult to reproduce the problem solving skills used by the nurse practitioners in this study for someone with no prior training. The frequency of the intervention is varied but not the intervention content - it is possible that another intervention would be more effective. This study was not designed to compare effectiveness of different interventions, but to determine the optimal frequency of an intervention that was thought to be optimal based on a previously published meta-analysis[41]. The study protocol did not address how often the subjects saw their primary care providers, the care provided by the primary care providers or counseling given in that setting.

This study seeks to assess the efficacy of varying frequencies of a highly structured nurse initiated telephonic intervention for the prevention of glycemic relapse. Prevention of glycemic relapse is a novel area in diabetes care that remains largely unstudied. By adjusting the frequency of the intervention, the optimal "dose" of intervention to maintain adequate glycemic control can be determined. This study will add to the fund of knowledge on longitudinal diabetes care.

\section{Competing interests}

The author(s) declare that they have no competing interests.

\section{Authors' contributions}

MMH participated in the statistical analysis and was the primary writer of the manuscript. AS participated in the study design and performed the statistical analysis. SM and LS assisted with study implementation, data acquisition and database management. AB, KW, EBK, RPG and DD assisted with study implementation and data acquisition. RS participated in study design and will perform economical analysis. TG assisted with statistical analysis. KC participated in study design, physical activity data and analysis of the physical activity data. RR, DS participated in study design. JWP assisted with study design and performed the intervention fidelity analysis. TAE conceived of the study, participated in the design, analysis, data management and helped draft the manuscript. All authors read the manuscript, provided editorial comments and approved the final manuscript.

\section{Acknowledgements}

The research was supported by the NIDDK RI8 DK 062258 and P60 DK 020593.

\section{References}

I. Harris MI, Flegal KM, Cowie CC, Eberhardt MS, Goldstein DE, Little RR, Wiedmeyer HM, Byrd-Holt DD: Prevalence of diabetes, impaired fasting glucose, and impaired glucose tolerance in U.S. adults. The Third National Health and Nutrition Examination Survey, 1988-1994. Diabetes Care 1998, 21:5। 8-524.

2. Roman SH, Harris MI: Management of diabetes mellitus from a public health perspective. Endocrinol Metab Clin North Am 1997, 26:443-474.

3. The effect of intensive treatment of diabetes on the development and progression of long-term complications in insulindependent diabetes mellitus. The Diabetes Control and Complications Trial Research Group. N Engl J Med 1993, 329:977-986.

4. U.K. prospective diabetes study 16. Overview of 6 years' therapy of type II diabetes: a progressive disease. U.K. Prospective Diabetes Study Group. Diabetes 1995, 44: I249-I 258.

5. Nathan DM, Cleary PA, Backlund JY, Genuth SM, Lachin JM, Orchard TJ, Raskin P, Zinman B: Intensive diabetes treatment and cardiovascular disease in patients with type I diabetes. N Engl J Med 2005, 353:2643-2653.

6. Gilmer TP, O'Connor PJ, Manning WG, Rush WA: The cost to health plans of poor glycemic control. Diabetes Care 1997, 20:1847-1853.

7. Harris MI: Noninsulin-dependent diabetes mellitus in black and white Americans. Diabetes Metab Rev 1990, 6:71-90.

8. Kenny SJ, Smith PJ, Goldschmid MG, Newman JM, Herman WH: Survey of physician practice behaviors related to diabetes mellitus in the U.S. Physician adherence to consensus recommendations. Diabetes Care 1993, 16:1507-1510.

9. Spann SJ, Nutting PA, Galliher JM, Peterson KA, Pavlik VN, Dickinson LM, Volk RJ: Management of type 2 diabetes in the primary care setting: a practice-based research network study. Ann Fam Med 2006, 4:23-31.

10. Gaede P, Vedel P, Larsen N, Jensen GVH, Parving HH, Pedersen O: Multifactorial Intervention and Cardiovascular Disease in Patients with Type 2 Diabetes 10.1056/NEJMoa02 I778. N Engl J Med 2003, 348:383-393.

II. Anderson RM, Funnell MM, Butler PM, Arnold MS, Fitzgerald JT, Feste CC: Patient empowerment. Results of a randomized controlled trial. Diabetes Care 1995, 18:943-949. 
12. Campbell EM, Redman S, Moffitt PS, Sanson-Fisher RW: The relative effectiveness of educational and behavioral instruction programs for patients with NIDDM: a randomized trial. Diabetes Educ 1996, 22:379-386.

13. Estey $A L$, Tan MH, Mann K: Follow-up intervention: its effect on compliance behavior to a diabetes regimen. Diabetes Educ 1990, I 6:29|-295.

14. Glasgow RE, La Chance PA, Toobert DJ, Brown J, Hampson SE, Riddle $M C$ : Long-term effects and costs of brief behavioural dietary intervention for patients with diabetes delivered from the medical office. Patient Educ Couns 1997, 32: I75-184.

15. Maxwell AE, Hunt IF, Bush MA: Effects of a social support group, as an adjunct to diabetes training, on metabolic control and psychosocial outcomes. Diabetes Educ 1992, 18:303-309.

16. Perry TL, Mann JI, Lewis-Barned NJ, Duncan AW, Waldron MA, Thompson C: Lifestyle intervention in people with insulindependent diabetes mellitus (IDDM). Eur J Clin Nutr 1997, 5 I:757-763.

17. Peters AL, Legorreta AP, Ossorio RC, Davidson MB: Quality of outpatient care provided to diabetic patients. A health maintenance organization experience. Diabetes Care 1996, 19:60 I-606.

18. Reichard $\mathrm{P}$, Toomingas B, Rosenqvist U: Changes in conceptions and attitudes during five years of intensified conventional insulin treatment in the Stockholm Diabetes Intervention Study (SDIS). Diabetes Educ 1994, 20:503-508.

19. Spiess K, Sachs G, Pietschmann P, Prager R: A program to reduce onset distress in unselected type I diabetic patients: effects on psychological variables and metabolic control. Eur J Endocrinol 1995, I32:580-586.

20. Tu KS, McDaniel G, Gay JT: Diabetes self-care knowledge, behaviors, and metabolic control of older adults--the effect of a posteducational follow-up program. Diabetes Educ 1993, 19:25-30.

21. Uusitupa M, Laitinen J, Siitonen O, Vanninen E, Pyorala K: The maintenance of improved metabolic control after intensified diet therapy in recent type 2 diabetes. Diabetes Res Clin Pract 1993, 19:227-238.

22. Uusitupa MI: Early lifestyle intervention in patients with noninsulin-dependent diabetes mellitus and impaired glucose tolerance. Ann Med 1996, 28:445-449.

23. Vanninen E, Uusitupa M, Siitonen O, Laitinen J, Lansimies E: Habitual physical activity, aerobic capacity and metabolic control in patients with newly-diagnosed type 2 (non-insulin-dependent) diabetes mellitus: effect of I-year diet and exercise intervention. Diabetologia 1992, 35:340-346.

24. Weinberger M, Kirkman MS, Samsa GP, Shortliffe EA, Landsman PB, Cowper PA, Simel DL, Feussner JR: A nurse-coordinated intervention for primary care patients with non-insulin-dependent diabetes mellitus: impact on glycemic control and health-related quality of life. J Gen Intern Med 1995, 10:59-66.

25. Wing RR, Blair E, Marcus M, Epstein LH, Harvey J: Year-long weight loss treatment for obese patients with type II diabetes: does including an intermittent very-low-calorie diet improve outcome? Am J Med 1994, 97:354-362.

26. Graber AL, Davidson P, Brown AW, McRae JR, Woolridge K: Dropout and relapse during diabetes care. Diabetes Care 1992, I 5: |477-1483.

27. Elasy TA, Graber AL, Wolff K, Brown A, Shintani A: Glycemic relapse after an intensive outpatient intervention for type 2 diabetes. Diabetes Care 2003, 26:1645-1646.

28. Turner RC, Cull CA, Frighi V, Holman RR: Glycemic control with diet, sulfonylurea, metformin, or insulin in patients with type 2 diabetes mellitus: progressive requirement for multiple therapies (UKPDS 49). UK Prospective Diabetes Study (UKPDS) Group. Jama 1999, 28 I:2005-2012.

29. Elasy TA, Ellis SE, Brown A, Pichert JW: A taxonomy for diabetes educational interventions. Patient Educ Couns 200I, 43: I II-127.

30. Miller WR, Rollnick S: Motivational Interviewing: Preparing People For Change. Second edition. New York, Guilford Publications; 2002:428.

31. Bellg AJ, Borrelli B, Resnick B, Hecht J, Minicucci DS, Ory M, Ogedegbe G, Orwig D, Ernst D, Czajkowski S: Enhancing treatment fidelity in health behavior change studies: best practices and recommendations from the NIH Behavior Change Consortium. Health Psychol 2004, 23:443-45I.
32. Dumas JE, Lynch AM, Laughlin JE, Phillips Smith E, Prinz RJ: Promoting intervention fidelity. Conceptual issues, methods, and preliminary results from the EARLY ALLIANCE prevention trial. Am J Prev Med 200I, 20:38-47.

33. Pichert JW, Stetson BA: Evaluating the treatment integrity of a continuing education program. J Nurs Staff Dev 1994, 10:75-80.

34. Chen KY, Sun M: Improving energy expenditure estimation by using a triaxial accelerometer. J Appl Physiol 1997, 83:2 I I2-2 I 22.

35. Radloff LS: The CES-D Scale: $\mathbf{A}$ self-report depression scale for research in the general population. Appl Psych Measurement 1977, I:385-401.

36. Horgren CT, Foster G: Cost Accounting: A Managerial Emphasis. 7th edition. Englewood Cliffs, NJ, Prentice Hall; I99I.

37. Perri MG, Shapiro RM, Ludwig WW, Twentyman CT, McAdoo WG: Maintenance strategies for the treatment of obesity: an evaluation of relapse prevention training and posttreatment contact by mail and telephone. J Consult Clin Psychol 1984, 52:404-4I3.

38. Baum JG, Clark HB, Sandler J: Preventing relapse in obesity through posttreatment maintenance systems: comparing the relative efficacy of two levels of therapist support. J Behav Med 1991, I4:287-302.

39. Marlatt GA: Taxonomy of high-risk situations for alcohol relapse: evolution and development of a cognitive-behavioral model. Addiction 1996, 91 Suppl:S37-49.

40. Baer JS, Marlatt GA: Maintenance of smoking cessation. Clin Chest Med 1991, I 2:793-800.

4I. Ellis SE, Speroff T, Dittus RS, Brown A, Pichert JW, Elasy TA: Diabetes patient education: a meta-analysis and meta-regression. Patient Education and Counseling 2004, 52:97- 105.
Publish with Biomed Central and every scientist can read your work free of charge

"BioMed Central will be the most significant development for disseminating the results of biomedical research in our lifetime. "

Sir Paul Nurse, Cancer Research UK

Your research papers will be:

- available free of charge to the entire biomedical community

- peer reviewed and published immediately upon acceptance

- cited in PubMed and archived on PubMed Central

- yours - you keep the copyright 